\title{
Dynamic Connectivity and Packet Propagation Delay in ALOHA Wireless Networks
}

\author{
Radha Krishna Ganti and Martin Haenggi \\ Department of Electrical Engineering \\ University of Notre Dame \\ Notre Dame, IN 46556, USA \\ \{rganti,mhaenggi\}@nd.edu
}

\begin{abstract}
We consider an ad hoc network which uses multihop and slotted ALOHA for its MAC contention. We then formulate the minimum time required for a packet to reach the destination from the origin. We define this delay as the minimum time required for a causal path to form between the source and destination. We derive the distributional properties of the connection time using ideas from first passage percolation and epidemic processes. We show that the delay scales linearly with the distance and also provide asymptotic results (w.r.t time) for the positions of the nodes which are able to connect to a transmitter located at the origin. We also provide simulation results to support the theoretical results.
\end{abstract}

Index Terms-Poisson point process, interference, first passage percolation, subadditive processes, epidemic processes.

\section{INTRODUCTION}

In a multihop ad hoc network, bits, frames or packets are transferred from a source to a destination in a multihop fashion with the help of intermediate nodes. This introduces a delay before a packet is delivered to the destination. For example, a five-hop route does not guarantee a delay of only five time slots. In a general setting, each node can connect to multiple nodes. So a large number of paths may form between the source and the destination. Each path may have taken a different time to form with the help of different intermediate nodes. Consider a network in which each node wants to transmit to its destination in a multihop fashion. In general in such a network, a relay node queues the packets from other nodes and its own packets and transmits them depending on some scheduling algorithm. If one introduces the concept of queues, the analysis of the system becomes extremely complicated because of the intricate dependencies between various nodes. In this paper we take a different approach. W are concerned only about the physical connections between nodes. That is, we do not care when a node $i$ transmits a particular packet to a node $j$ (depends on the scheduler), but we analyze when a (physical) connection (maybe over multiple hops) is formed between the nodes $i$ and $j$. This delay gives a lower bound on the delay with any queueing scheduler in place.

We assume that the nodes are distributed as a Poisson point process (PPP) on the plane. Each node in a time slot decides to transmit or receive using ALOHA. Any transmitting node can connect to all receiving nodes whose SINR threshold is met. Since at each time instant, the transmit and receive nodes change, we have a dynamic connectivity graph. We analyze the time required for a causal path to form between a source and a destination node. The system model is made precise in Section II.
This problem is similar in flavor to the problem of First Passage Percolation (FPP)[10], [8], [1]. This process of dynamic connectivity also resembles a simple epidemic process[5], [13], [14] on an Euclidean domain. In a spatial epidemic process, a infected individual infects a certain (maybe random) neighboring population and this process continues until the complete population is infected. They analyze the time of spread of the epidemic process. We draw many ideas from this theory of epidemic process and FPP. The main difference between an epidemic process and the process we consider is that the spread (of packets) depends on the entire population (due to interference) and is not independent from a node to node. In [4], the authors analyze the latency for a message to propagate in a sensor network using similar tools. Their model does not consider interference and their model allows them to use Kingman's subadditive ergodic theorem [11] while ours does not. They consider a Boolean connectivity model with random weighed edges and derive the properties of first passage paths on the weighted graph.

In Section II, we introduce the system model. In Section III, we derive the properties of the delay, the average number of paths between a source and destination. In Section IV we give limit theorems for the delay and the broadcast region. We specifically show that the delay increases linearly with increasing transmit-receive distance or equivalently that the propagation speed is constant, i.e., the distance of nodes which can connect to the origin scales linearly with time.

\section{SySTEM MODEL}

The system consists of nodes distributed as a Poisson point process (PPP) $\Delta$ of intensity $\lambda$ on the plane. Each node can either transmit or receive. This is decided by slotted ALOHA with parameter $p$. So at each time slot $k$, each node decides to either transmit or receive with probability $p$ and $q=1-$ $p$ respectively. Let the transmitting set at time instant $k$, be denoted by $\phi(k)$ and the receiver set by $\psi(k)$. So we have $\Delta=$ $\phi(k) \cup \psi(k)$. One can alternatively think of the whole setup as a marked point process, with each mark being an discrete Bernoulli process (each time instant of the process defines whether a node is a transmitter or receiver). More formally the point process we consider is on the set $\mathbb{R}^{2} \times S$, where $S=$ $\left\{\left(a_{1}, a_{2}, a_{3}, \ldots\right): a_{i} \in\{0,1\}\right\}$, the set of all 0,1 sequences.

We consider the following transmission models:

1) Disk model (Protocol model): A transmitter located at $x$, can successfully transmit to a receiver located at $y$ if $x \in B(y, a)$ and $\{\phi(k) \backslash x\} \cap B(y, b)=\emptyset, b>a$.

2) Interference model (Physical model): In this model, the power received by a receiver located at $z$ due to a 
transmitter at $x$ is modeled as $h_{x z}\|x-z\|^{-\alpha}$, where $h_{x z}$ denotes the square of the Rayleigh fading coefficient. The sum-power at point $z$ on the plane is given by

$$
I_{\phi(k)}(z)=\sum_{x \in \phi(k)} h_{x z}\|x-z\|^{-\alpha}
$$

We say that the communication from a transmitter at location $x \in \phi(k)$ to a receiver situated at $\psi(k)$ is successful [2] if and only if

$$
\frac{h_{x z}\|x-z\|^{-\alpha}}{I_{\phi(k) \backslash\{x\}}(z)} \geq \beta
$$

where $h_{x z}$ is the fading coefficient between node $x$ and receiver $z$.

At each time instant $k>0$, we form a directed graph $g(k)=$ $\left(\Delta, E_{k}\right)$, whose vertex set is $\Delta$ and edge set is $E_{k}$. A directed edge is placed between a node in the transmit set $\phi(k)$ and a node in $\psi(k)$ if (2) (or the condition for model one) is satisfied. Also each edge has a time stamp as its weight. Let $G(m, n)$ denote the weighted directed multigraph (multiple edges with different time stamps allowed between two vertices) formed between times $m$ and $n>m$, i.e.,

$$
G(m, n)=\left(\Delta, \cup_{k=n}^{m} E_{k}\right)
$$

A sequence of directed edges, is said to be a causal path if the weights form an strictly increasing sequence. From now, by a path we mean a causal path. In this paper we study the properties (averaged over the realizations of $\Delta$ ) of $G(0, n)$ and the time required for a path to form between two points and their dependence on the system parameters $(a, b, \lambda, \beta, \alpha, p)$. The edge set for the interference model is given by

$$
E_{k}=\left\{(x, z) ; \frac{h_{x z}\|x-z\|^{-\alpha}}{I_{\phi(k) \backslash\{x\}}(z)} \geq \beta, x \in \phi(k), z \in \psi(k)\right\}
$$

where $(x, z)$ represents a directed edge from $x$ to $z$. We assume that the interference at different time instants is independent. This is a reasonable assumptions since at each time instant, the transmitter set is changing due to ALOHA and there is fading. More precisely we assume the following, $\forall m \neq n$,

$$
E\left[1_{E_{m}}(a, b) 1_{E_{n}}(c, d)\right]=E\left[1_{E_{m}}(a, b)\right] E\left[1_{E_{n}}(c, d)\right]
$$

where the expectation is with respect to fading, ALOHA and the point process $\Delta$.

Notation: $B(x, r)$ denotes an open ball of radius $r$ around the point $x$. Let $e_{1}=(1,0)$ and $e_{2}=(0,1)$ denote the unit vectors in the directions of the coordinate axes. We will use $\stackrel{d}{=}$ to denote equality in distribution.

\section{Properties of the AVERage $G(0, n)$.}

1) Average in and out degree: For the interference model, the node degree for an arbitarly chosen receiving node (chosen at origin) for a single time instant $k$ given that the node at the origin is listening is

$$
d_{I}(k)=\sum_{x \in \phi(k)} 1(x \text { able to connect to origin }) .
$$

So by the Campbell-Mecke theorem [15], we have

$$
\begin{aligned}
E\left[d_{I}(k)\right] & =\lambda p \int_{\mathbb{R}^{2}} \exp \left(-\lambda p\|x\|^{2} \beta^{2 / \alpha} C(\alpha)\right) d x \\
& =\pi \beta^{-2 / \alpha} C(\alpha)^{-1}
\end{aligned}
$$

where $C(\alpha)=2 \pi^{2} /(\alpha \sin (2 \pi / \alpha))$. So the average in degree of a typical node in an interference model is

$$
E\left[D_{I}(n)\right]=n \pi(1-p) \beta^{-2 / \alpha} C(\alpha)^{-1}
$$

Following similar lines, the average in degree of the disk model is given by $n \pi \lambda p(1-p) a^{2} \exp \left(-\lambda p \pi b^{2}\right)$. In the disk model, observe that a transmitter can conect to multiple receivers, while a receiver can listen to only one transmitter. We observe that the average in degree increases linearly with time $n$. Also we observe that the in degree of the interference model is independent of $\lambda$ (this is partly because we choose the path-loss model to be $\|x\|^{-\alpha}$ ), while the average in degree of the disk model tends to zero as $\lambda \rightarrow \infty$. Also we can expect a Gaussian distribution as $n$ becomes large, since the in degree is the sum of iid random variables. Using similar arguments, one can show that the average out degree for the interference model is given by

$$
E\left[D_{o}(n)\right]=n \pi(1-p) \beta^{-2 / \alpha} C(\alpha)^{-1} .
$$

For a disk model the average out-degree is given by $n \pi \lambda p(1-$ p) $a^{2} \exp \left(-\lambda p \pi b^{2}\right)$. So the average in and out-degrees match which is intuitive.

2) Average number of paths between o and $x$ by time $n$ : Add two points, one at the origin $o$ and another at location $x$ to $\Delta$. Let $A(k), 1 \leq k \leq n$ denote the adjacency matrix of the graph $g(k)$, with the nodes ordered with respect to the distance from origin. Then $A_{i j}(k)=\left\{1_{E_{k}}\left(x_{i}, x_{j}\right)\right\}$. We observe that $A_{i j}(k), 1 \leq k \leq n$, is iid distributed for all $i, j$.

Lemma 1: With the assumption (3), the average number of paths between $o$ and $x$ in an interference model is given by $E\left[N_{n}(x)\right]=$

$$
\sum_{k=1}^{n}\left(\begin{array}{l}
n \\
k
\end{array}\right) \frac{p q}{k}\left(\frac{\pi q}{\beta^{2 / \alpha} C(\alpha)}\right)^{k-1} \exp \left(-\frac{p}{k} \lambda\|x\|^{2} \beta^{2 / \alpha} C(\alpha)\right)
$$

Proof: Let $\mathcal{A}^{n}=\prod_{i=1}^{n}(I+A(i))$. So the total number of edges $N_{n}(x)$ will be the entry in the row corresponding to the origin and the column of $x$ in $\mathcal{A}^{n} . N_{n}(x)=\sum_{k=1}^{n} \tilde{N}_{n}^{k}(x)$ where $\tilde{N}_{n}^{k}(x)$ denote the number of $k$ length paths between $o$ and $x$ by time $n$. We first evaluate $E\left[\tilde{N}_{n}^{k}(x)\right]$. From the matrix $\mathcal{A}^{n}$ and the iid property of $A(k)$, we have, $E\left[\tilde{N}_{n}^{k}(x)\right]=$

$$
\left(\begin{array}{l}
n \\
k
\end{array}\right) E\left[\sum_{x_{1} \ldots x_{k-1} \in \Delta \backslash\{o, x\}}^{\neq} \prod_{m=0}^{k-1} 1_{E_{m}}\left(x_{m}, x_{m+1}\right)\right]
$$

where $x_{0}=o$ and $x_{k}=x$ and $\sum^{\neq}$denotes summation over disjoint points. So we are not counting loops. Using (3) and the Campbell-Mecke theorem we have $E\left[\tilde{N}_{n}^{k}(x)\right]$

$$
\begin{aligned}
= & \left(\begin{array}{l}
n \\
k
\end{array}\right) \lambda^{k-1} \int \prod_{m=0}^{k-1} \mathbb{P}\left(\left(x_{m}, x_{m+1}\right) \in E_{1}\right) d x_{1} \ldots d x_{k-1} \\
= & \left(\begin{array}{l}
n \\
k
\end{array}\right) \lambda^{k-1}(p q)^{k} . \\
& \int \exp \left(-\lambda p \beta^{2 / \alpha} C(\alpha) \sum_{i=0}^{k}\left\|x_{i}-x_{i+1}\right\|^{2}\right) d x_{1} \ldots d x_{k-1}
\end{aligned}
$$

We have the following observations regarding the average number of paths between $o$ and $x$ in $n$ time instants.

1) Observe that for large $\|x\|$ the main contributors are 
$E\left[\tilde{N}_{n}^{k}(x)\right], k$ large, i.e., multihop routing becomes important as $\|x\|$ increases.

2) For a large $\|x\|$, to have a good average number of paths, $n$ should scale approximately like $\|x\|^{2}$.

3) One can think of $E\left[N_{n}(x)\right]$ as the average path diversity that is offered to a packet.

Corollary 1: Let $\|x\|^{2}$ scale as $n^{\delta}$. Consider the average number of paths as $n \rightarrow \infty$. We then have

$$
\lim _{n \rightarrow \infty} E\left[N_{n}\left(n^{\delta}\right)\right]= \begin{cases}\infty, & \delta<1 \\ \infty & \delta \in[1,2), \frac{\pi q}{\beta^{2 / \alpha} C(\alpha)}>\frac{1}{2} \\ 0 & \delta>2\end{cases}
$$

\section{SCALING LAWS FOR DELAY AND PROPAGATION SPEED}

In this section we show that the propagation delay increases linearly with the transmit-receive distance. One can infer from the result, that one does not require full connectivity in a single instant. One can therefore relax the condition of a giant connected component in an interference network [3], since the time required for connection only scales linearly with time. Also most of the results in this section do not heavily rely on the particular connectivity models we considered. For $x, y \in \Delta$, we denote the time for a connection to form between $x$ and $y$ as

$$
T(x, y)=\min \{k: G(0, k) \text { has a path from } x \text { and } y\} \text {. }
$$

For general $x, y \in \mathbb{R}^{2}, T(x, y)=T\left(x^{*}, y^{*}\right)$ where $x^{*}$ (resp. $\left.y^{*}\right)$ is the point in $\Delta$ closest to $x$ (resp. $y$ ), with some fixed deterministic rule for breaking ties (no ties almost surely). We similarly define

$T_{n}(x, y)=\min \{k-n: G(n, k)$ has a path from $x$ and $y\}$.

Let

$$
\tilde{B}_{t}=\{x: T(o, x) \leq t\}
$$

denote the set of points which can be reached from the origin by time $t$. We denote the farthest distance reached by time $t$ as

$$
D_{t}=\sup \{\|x\|: T(o, x) \leq t\}
$$

The evolution of the graph $G(0, n)$ is similar to the growth of epidemic on the plane and one can relate this problem to the theory of Markovian contact process [14] which was used to analyze the growth of epidemics. From the definition of $T(o, m)$, we observe that

$$
T(o, m) \leq T(o, n)+T_{T(o, n)}(n, m) .
$$

We also have that $T_{T(o, n)}(n, m) \stackrel{d}{=} T(n, m)$ from the way the graph progress is defined. Also if we just use a scalar like $n$ for one of the arguments of $T(x, y)$, it should be interpreted as $n e_{1}$.

Lemma 2: The time constant defined by

$$
\mu=\lim _{n \rightarrow \infty} \frac{E T(o, n)}{n}
$$

exists and $\mu \in[0, \infty)$

Proof: From (4), we have

$$
T(o, m+n) \leq T(o, m)+T_{T(o, m)}(m, m+n) .
$$

From the definition of the graph, $E_{k}$ does not depend on $E_{i}, i<k$. Hence we have that $T_{T(o, m)}(m, m+n)$ has the same distribution as $T(m, m+n)$. Also from the invariance of the point process $\Delta$, we have $T(m, m+n) \stackrel{d}{=} T(o, n)$. Taking expectation of (5), we have

$$
E T(o, m+n)<E T(o, m)+E T(o, n)
$$

Hence the result follows from the basic properties of the subadditive sequences. Also $\mu=\inf _{n \geq 1} \operatorname{ET}\left(o, n e_{1}\right) / n$.

Consistent with the terminology of first passage percolation (FPP) we will call $\mu$ the time constant of the process. Observe that (5) resembles the triangle inequality (specially if $T_{T(o, m)}(m, m+n)$ was $\left.T(m, n)\right)$. This resembles a pseudo-metric and is the reason that the shortest paths in FPP are called geodesics. Since we do not have $T(o, n+$ $m) \leq T(o, n)+T(o, m)$, Kingman's subadditive ergodic theorem [11] cannot be directly applied to (4). But since $T_{T(0, n)}(n, m) \stackrel{d}{=} T(n, m)$, we may hope that such a result would hold true. In the next lemma, we prove that this is indeed the case.

Lemma 3: Let $\mu$ be the time constant of the process,

$$
\frac{T(o, n)}{n} \longrightarrow \mu
$$

where the convergence is in $L^{2}$ and hence in probability.

Proof: From (4), and $T_{T(o, m)}(m, m+n) \stackrel{d}{=} T(o, n)$ and the fact that $T_{T(o, m)}(m, m+n)$ is independent of $T(o, m)$ (because of assumption (3)), we have

$$
F_{n+m}(x) \geq\left(F_{n} * F_{m}\right)(x)
$$

where $F_{n}$ is the CDF of $T(o, n) . E\left(T(o, n)^{2}\right)<\infty$ follows from Lemma 6 . So we have a superconvolutive sequence and hence by Kesten's lemma [16], [7], (6),[12, P. 120] holds ${ }^{1}$.

This result shows that with high probability, the delay required for a packet propagation scales linearly with distance. The next result concerns the maximum distance a packet travels by time $t$.

Lemma 4: The velocity of information propagation converges, i.e.

$$
\frac{D_{n}}{n} \rightarrow \mu_{1}
$$

$n \in \mathbb{N}$, with probability one. Also $\mu_{1}=\frac{1}{\mu} \in(0, \infty)$.

Proof: We have

$$
D_{m+n} \geq D_{m}+\tilde{D}_{m n}
$$

where $\tilde{D}_{m n}$ is the farthest distance in $n$ time steps starting from the point which achieved $D_{m}$. We observe that $\tilde{D}_{m n} \stackrel{d}{=}$ $D_{n}$. Hence from (8), we have that $D_{n}$ forms a subconvolutive sequence and hence $-D_{n}$ forms a superconvolutive sequence. So if we show $E\left[D_{1}^{2}\right]<\infty$, by Kesten's lemma $L^{2}$ convergence follows. We have

$$
\mathbb{P}\left(D_{1}<x\right)
$$

$=\mathbb{P}($ The origin is not able to connect

to any node at distance greater than $x$ )

$$
\begin{aligned}
& \stackrel{(a)}{\geq} \exp \left[-\lambda(1-p) \int_{B(0, x)^{c}} \exp \left(-\lambda p \beta^{2 / \alpha} C(\alpha)\|y\|^{2}\right) d y\right] \\
& =\exp \left[-(1-p) p^{-1} \beta^{-2 / \alpha} C(\alpha)^{-1} e^{-\lambda p \beta^{2 / \alpha} C(\alpha) x^{2}}\right]
\end{aligned}
$$

\footnotetext{
${ }^{1}$ To prove the a.e convergence using Kesten's lemma, we require that $T(0, n)$ be a monotone sequence, which is not true in our case.
} 


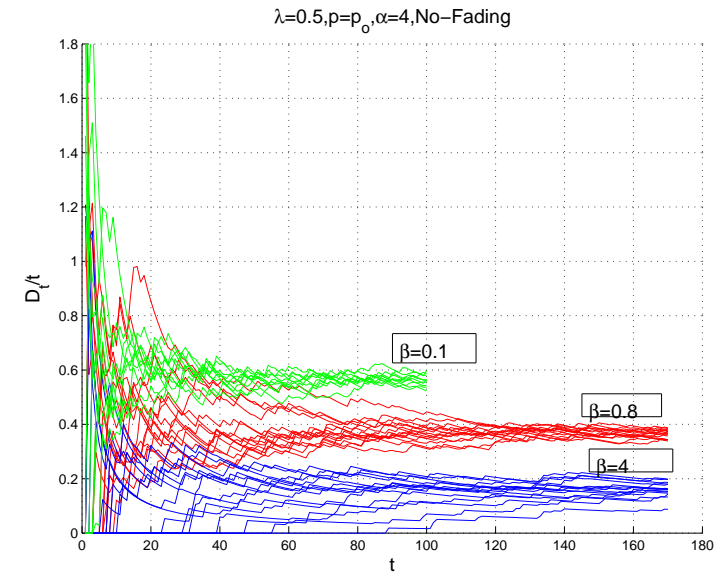

Figure 1. Illustration of the a.e. convergence of $D_{t} / t$

For $(a)$ see [6, Sec 2.A]. From (10), we observe that the tail probability of $D_{1}$ decays exponentially fast and hence has a finite second moment. For the disk model we have $D_{1}<b$ and hence has a finite second moment. Since the sequence $D_{n}$ is also monotonic, we have convergence with probability one. We also have $E\left[D_{n}\right] / n>E\left[D_{1}\right]>0$. Using Lemma 7, one can also deduce $\mu_{1}=1 / \mu$ and from Lemma 5 , we have $\mu_{1}<\infty$ for the interference and the disk model.

Lemma 5: For the interference and disk models ${ }^{2} \mu>0$.

Proof: The basic idea is to use the conflict that large hops implies fewer hops but also imply a smaller probability of connection and hence more time to connect. Smaller hops means smaller time of connection (better probability) but a larger number of hops. We first show that $\mu>0$ for the disk model we have considered. We have that

$$
\mathbb{P}\left(T(o, n)<\frac{n}{a}\right)=0
$$

since the maximum distance covered in a single time slot is $a$. Hence we have that $E[T(o, n) / n]>1 / a$. This implies $\mu>1 / a>0$.

We will show that

$$
\mathbb{P}(T(o, n)<c n) \rightarrow 0
$$

as $n \rightarrow \infty$ for some positive $c$. This implies $E[T(o, n) / n]>$ $c$ for some $c>0$. For the sake of convenience let $c n$ be identified with $\lceil c n\rceil$. So to evaluate the event $\{T(o, n) \leq c n\}$, we consider only those paths which have a maximum of $c n$ hops. By the union bound we have

$$
\mathbb{P}(T(o, n)<c n) \leq \sum_{i=1}^{c n} p_{i}
$$

where $p_{i}=\mathbb{P}(T(o, n)<c n \mid$ we reached $n$ from $o$ in $i$ hops $)$. The time to any single direct hop between two nodes $o$ and $x$ is a geometric random variable with parameter $\nu(x)=$ $p(1-p) \exp \left(-c_{1}\|x\|^{2}\right)$, where $c_{1}=\lambda p \beta^{2 / \alpha} C(\alpha)$. So the times to form the individual hops in a $k$ hop path between $o, x_{1}, x_{2} \ldots, x_{k-1}, n$ are a series of geometric random variables $t_{i}$ with parameters $v\left(x_{i-1}-x_{i}\right)$ (they are independent because they occur in different time slots. See (3)). Let $\eta>0$.

\footnotetext{
${ }^{2}$ In general we conjecture if the probability of connectivity decreases exponentially, $\mu>0$
}

So we have

$$
\begin{aligned}
p_{k} \leq & \mathbb{P}\left(\sum_{i=1}^{k} t_{i}<c n\right) \\
\stackrel{(a)}{\leq} & \exp (\eta c n) \frac{1}{(\exp (\eta)-1)^{k}} \prod_{i=1}^{k} v\left(x_{i-1}-x_{i}\right) \\
= & \exp (\eta c n)\left(\frac{p(1-p)}{\exp (\eta)-1}\right)^{k} \exp \left(-c_{1}\left(\|x\|_{1}^{2}\right.\right. \\
& \left.\left.+\left\|x_{2}-x_{1}\right\|^{2}+\ldots+\left\|x_{k-1}-n\right\|^{2}\right)\right) \\
\stackrel{(b)}{\leq} & \exp (\eta c n)\left(\frac{p(1-p)}{\exp (\eta)-1}\right)^{k} \exp \left(-c_{1} \frac{n^{2}}{k}\right)
\end{aligned}
$$

(a) follows from the observation ${ }^{3}$ and $(b)$ follows from the fact that the minimum value of $\|x\|_{1}^{2}+\left\|x_{2}-x_{1}\right\|^{2}+\ldots+$ $\left\|x_{k-1}-n\right\|^{2}$ is $n^{2} / k$. So from (11), we have $\mathbb{P}(T(o, n)<c n)$

$$
\begin{aligned}
& \leq \sum_{k=1}^{c n} \exp (\eta c n+\eta k) p^{k}(1-p)^{k} \exp \left(-c_{1} \frac{n^{2}}{k}\right) \\
& \stackrel{(a)}{\leq} \quad c n \exp (\eta c n) \exp \left(-\frac{c_{1}}{c} n\right)
\end{aligned}
$$

where $(a)$ follows by choosing $\eta^{\prime}$ such that $p(1-p) /\left(\exp \left(\eta^{\prime}\right)-\right.$ $1)<1$ and using $k=c n$ for all the terms. The right hand side goes to 0 if $c<\sqrt{c_{1} / \eta^{\prime}}$. Hence we have $E[T(0, n) / n]>c$ which implies $\mu>c$.

We now analyze the properties of the time required for a direct connection to form between $o$ and $x$. For both the models, the time required is a geometric distribution, i.e.,

$\mathbb{P}$ (Time taken for the first direct connection

$$
\text { between } o, x=n)=(1-\eta)^{n-1} \eta
$$

1) Interference model:

$$
\eta=p(1-p) \exp \left(-\lambda p\|x\|^{2} \beta^{2 / \alpha} C(\alpha)\right) \text {. }
$$

2) Disk model: This can happen iff $\|x\|<a$ and $\eta=p(1-p) \exp \left(-\lambda p \pi b^{2}\right)$.

So we have the following trivial bound, $E[T(0, n)]<n \eta^{-1}$. From this bound, a good value of $p$ to decrease the average delay is $p \approx\left(\beta^{-2 / \alpha} C(\alpha)^{-1}\right) / 2$.

Lemma 6: The tail probability of $T(0, n)$ when $\lambda$ is large (so that there is a node with high probability near each integer point) can be bounded as

$$
\mathbb{P}(T(0, n)>k) \leq I_{1-\eta}(k+1, n)
$$

where $I_{\eta}(n, k+1)$ is the regularized beta function.

Proof: We have

$$
T(0, n) \leq \sum_{i=1}^{n} T_{T(0, n-1)}(n-1, n)
$$

where $T_{T(0, n-1)}(i-1, i)$ are iid. We also have $T_{T(0, i-1)}(i-$ $1, i)<t_{i}$ where

$t_{i}=$ Time taken for the first direct connection between $i-1, i$

${ }^{3}$ If $t_{1} \ldots t_{i}$ iid geometric random variables with parameters $p_{i}$, then

$$
\mathbb{P}\left(\sum_{i=1}^{k} t_{i}<a\right) \leq \exp (\eta a)\left(\frac{e^{-\eta}}{1-e^{-\eta}}\right)^{k} \prod_{i=1}^{k} p_{i}
$$

for any $\eta>0$. 


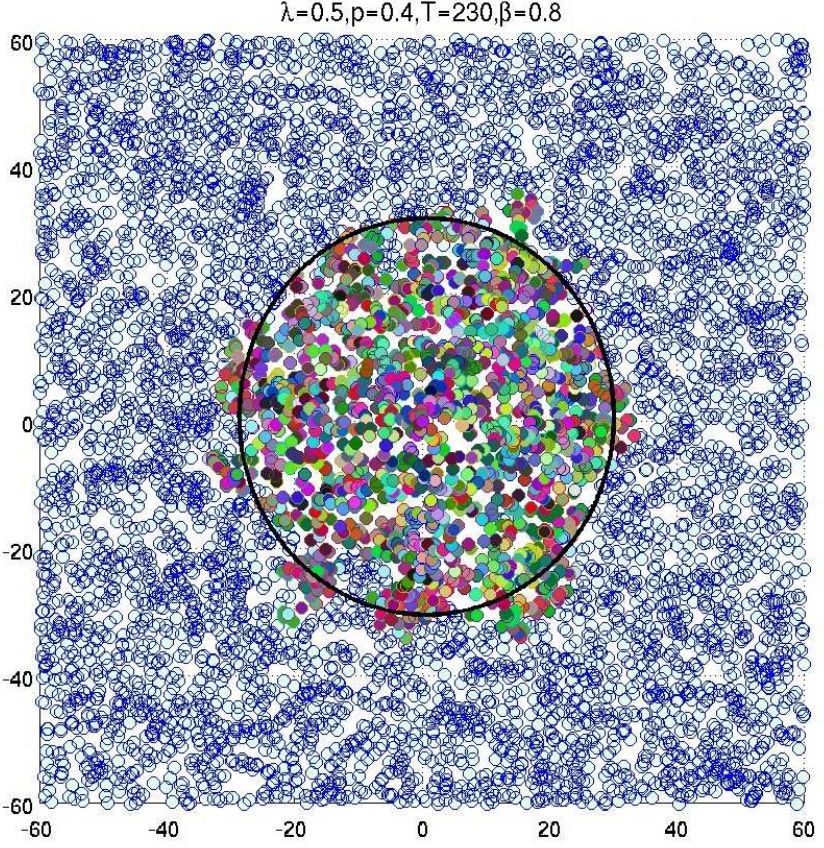

Figure 2. Illustration of the shape theorem

$t_{i}$ are iid and geometric distributed with parameter $\eta$. Hence we have

$$
\begin{aligned}
\mathbb{P}(T(0, n)>k) & \leq \mathbb{P}\left(\sum_{i=1}^{n} t_{i}>k\right) \\
& \stackrel{(a)}{=} 1-I_{\eta}(n, k+1) \\
& =I_{1-\eta}(k+1, n)
\end{aligned}
$$

(a) follows from the fact that the sum of geometric random variables follows a negative binomial distribution.

Instead of using the properties of the binomial random variables in (12), we can use the central limit theorem to derive: there exists three constants $c_{1}, c_{2}$ and $c_{3}$ such that

$$
\mathbb{P}\left(T(0, n)>c_{1} n\right) \leq c_{2} \exp \left(-c_{3} n\right)
$$

and the constants do not depend on $n$. By translation invariance and isotropy of the PPP, we have

$$
\mathbb{P}\left(T(x, y)>c_{1}\|x-y\|\right) \leq c_{2} \exp \left(-c_{3}\|x-y\|\right)
$$

Let $\tilde{B}_{t}=\{x: T(o, x) \leq t\}$, i.e., the set of points reachable by time $t$. We prove the convergence of $\tilde{B}_{t} / t$ to a fixed set $B\left(o, \mu^{-1}\right)$ (shape theorem). In (6), we considered the time along $o$ and $n$ along the x-axis. By the isotropy of the point process, and the process, we have that for any $e_{\theta} \in S^{1}$, $T\left(0, n e_{\theta}\right) / n \rightarrow \mu$ in $L^{2}$. The next two theorems follow closely the technique used in [9] to prove the shape theorem for Euclidean first passage percolation. For a random variable $X$ let $|X|_{2}$ denote the $L_{2}$ norm $\sqrt{E\left(X^{2}\right)}$. We present the next lemma without a proof because of space constraints.

Lemma 7: We have $\lim _{\|x\| \rightarrow \infty} T(o, x) /\|x\| \rightarrow \mu$ in $L^{2}$.

Note that we have replaced the integer index with a continuous variable over all directions.

Theorem 1: (Shape theorem) When $\mu>0, \tilde{B}_{t} / t$ converges in probability to $B\left(o, \mu^{-1}\right)$. More precisely $\forall \delta>0, \epsilon>0$, $\exists t_{0}$ such that

$\mathbb{P}\left(B\left(o,\left(\mu^{-1}-\epsilon\right)\right) \subset \frac{\tilde{B}_{t}}{t} \subset B\left(o,\left(\mu^{-1}+\epsilon\right)\right)\right)>1-\delta, \quad \forall t>t_{0}$

Proof: Follows from Lemma 7. The proof is similar to [9, Pg 10] replacing the statements of a.e. to "in probability".

\section{CONCLUSIONS}

In this paper we have introduced the concept of the time taken for a physical path to form between a transmitter and a receiver in a multihop ALOHA network. We have showed that this time scales linearly with the transmit receive pair distance. This in some sense implies that every node can be accessed in a time that is linear with the distance. So in a route discovery flooding algorithm, the time to find the route scales linearly with the diameter of the network. We also showed that the nodes which are able to connect to a node at the origin by a certain time are located in a circle of radius that scales linearly with time.

\section{ACKNOWLEDGEMENT}

The support of the NSF (Grants CNS 044769 and CCF 728763) and the DARPA/IPTO IT-MANET program (Award W911NF-07-1-0028) is gratefully acknowledged.

\section{REFERENCES}

[1] D. Aldous and JM Steele, Probability on Discrete Structures (Encyclopaedia of Mathematical Sciences vol 110) ed H Kesten, (2003).

[2] Francois Baccelli, B. Blaszczyszyn, and P. Muhlethaler, An ALOHA protocol for multihop mobile wireless networks, IEEE Transactions on Information Theory (2006), no. 2.

[3] O. Dousse, M. Franceschetti, N. Macris, R. Meester, and P. Thiran, Percolation in the signal to interference ratio graph, J. Appl. Prob 43 (2006), 552-562.

[4] O. Dousse, P. Mannersalo, and P. Thiran, Latency of wireless sensor networks with uncoordinated power saving mechanisms, Proceedings of the 5th ACM international symposium on Mobile ad hoc networking and computing (2004), 109-120.

[5] Rick Durrett, Stochastic spatial models, Siam Review 41 (1999), 677718.

[6] Radha Krishna Ganti and M. Haenggi, Single-hop connectivity in interference-limited hybrid wireless networks, ISIT 2007 (Nice, France), 2007.

[7] JM Hammersley, Postulates for Subadditive Processes, The Annals of Probability 2 (1974), no. 4, 652-680.

[8] JM Hammersley and DJA Welsh, First-passage percolation, subadditive processes, stochastic networks, and generalized renewal theory, Bernoulli-Bayes-Laplace Anniversary Volume (1965), 61-110.

[9] C.D. Howard and C.M. Newman, Euclidean models of first-passage percolation, Probability Theory and Related Fields 108 (1997), no. 2, 153-170.

[10] H. Kesten, Aspects of first passage percolation, Lecture Notes in Math 1180 (1986), 125-264.

[11] JFC Kingman, Subadditive Ergodic Theory, The Annals of Probability 1 (1973), no. 6, 883-899.

[12] Subadditive Processes, Ec. d'Ete Probab. Saint-Flour V- (1975).

[13] D. Mollison, Spatial Contact Models for Ecological and Epidemic Spread, Journal of the Royal Statistical Society. Series B (Methodological) 39 (1977), no. 3, 283-326.

[14] Markovian Contact Processes, Advances in Applied Probability 10 (1978), no. 1, 85-108.

[15] Dietrich Stoyan, Wilfrid S. Kendall, and Joseph Mecke, Stochastic geometry and its applications, second ed., Wiley series in probability and mathematical statistics, Wiley, New York, 1995.

[16] J.C. Wierman, The Front Velocity of the Simple Epidemic, vol. 16, JSTOR, 1979. 